\title{
The factor-ordering problem must be regulated
}

\author{
N. C. Tsamis \\ CERN Theory Group, CH-1211 Genève 23, Switzerland \\ R. P. Woodard* \\ Department of Physics, University of California, Santa Barbara, California 93106
}

(Received 30 January 1987)

\begin{abstract}
Three points are stressed concerning the factor-ordering problem of canonical quantum gravity. First, the problem is real; dimensional regularization in the covariant formalism permits us to systematically ignore it, but does not provide a resolution. Second, no resolution of the problem is possible until the naive constraint algebra is first regulated to remove divergences arising from singular operator products. This point is underlined with an example from the literature. Third, we argue that whatever regulation technique is employed should respect general coordinate invariance or some analog of it. Although the traditional variables of canonical quantum gravity are used, our conclusions apply with equal force to the new variables proposed by Ashtekar.
\end{abstract}

\section{INTRODUCTION}

In the hierarchy of problems connected with the quantization of gravity, our inability to deal with ultraviolet divergences ${ }^{1}$ and our failure to explain the smallness of the cosmological constant relative to other mass scales $^{2}$ rank highest. Both problems show up in the covariant formalism which has become ubiquitous since the advent of dimensional regularization ${ }^{3}$ and the background-field method. ${ }^{4}$ Far down the list of quantum gravity disasters are the difficulties associated with the less popular canonical formalism. ${ }^{5}$ Yet although canonical problems are generally conceded to be less urgent than their covariant relatives, they are real and a small trickle of effort has been devoted over the years to their resolution. Our contribution concerns what is known as the "factor-ordering problem."

This is not the usual issue of how to arrange noncommuting operators in the naive Hamiltonian, rather it consists of ordering the gravitational constraints so that they satisfy a condition known as "Dirac consistency." We will define what this means more precisely in Sec. II but the basic idea is simple enough to sketch out. Consider a constrained Hamiltonian system with coordinates $q$, momenta $p$, and constraints $\phi_{A}(p, q)=0$. Owing to the presence of constraints not every wave function $\psi(q)$ corresponds to a valid state-quite apart from any question of normalizability. The physical subspace of states is defined by the condition that its elements are annihilated by the constraints:

$$
\phi_{A}(p, q)|\psi\rangle=0 \text {. }
$$

Obviously the consecutive action of two constraints also gives zero and hence so does the commutator

$$
\left[\phi_{A}, \phi_{B}\right]|\psi\rangle=0 \text {. }
$$

Since relation (1.2) must hold for every state in the physical subspace, the commutator of two constraints must itself be a linear combination of constraints

$$
\left[\phi_{A}, \phi_{B}\right]=C_{A B C}(p, q) \phi_{C} .
$$

The combination coefficients $C_{A B C}(p, q)$ may themselves be operators but they must stand to the left of the constraints. Failure to satisfy relation (1.3) either means that there is a missing constraint or that the physical subspace consists of the state $\psi=0 .^{6}$ Note that although Dirac consistency is a necessary condition for the physical subspace to be nontrivial, it can, in principle, be studied in the strong operator sense. This is significant because one of the many things we do not know how to do very precisely in quantum gravity is to take inner products.

The above mathematics aside, there are two physical reasons to care about Dirac consistency: (1) it ensures that general coordinate invariance, which is no longer manifest once canonical methods have been employed, is present classically and survives quantization; ${ }^{7,8}$ (2) it may impose very strong restrictions on otherwise undetermined parameters in the quantum theory. Regarding the first point, it is quite possible that the functional integrals of the covariant formalism do not really represent inner products of states which obey the Wheeler-DeWitt equations. The phenomenon would show up as dependence upon the choice of residual gauge conditions (the usual Ward identities only prove independence of the volume gauge). Saying that the predictions of a theory depend upon the gauge is just another way of labeling the theory as nonsense. Thus Dirac consistency is a necessary condition for the covariant formalism to actually mean something.

As an example of the second point, note that enforcing a very similar condition in the theory of bosonic strings is responsible for selecting 26 as the only acceptable dimension for spacetime. ${ }^{9}$ There are a number of naively unconstrained parameters in quantum gravitythe dimension of spacetime, the value of the cosmological constant, the number and type of matter fields, and 
the internal matter-field symmetry group. Might it not be that enforcing Dirac consistency can tell us something about them? The answer is that we simply do not know. Though the classical constraints have long been known to close under Poisson bracketing, ${ }^{7}$ no one has been able to obtain a clear result on the quantum level.

Not that there has been any dearth of efforts. Dirac cast the constraints in their present form in $1958,{ }^{10}$ however, his 1959 approach to quantization ${ }^{11}$ was unsatisfactory in that it merely transformed the problem to one of defining suitable Dirac brackets. Similar results were obtained by Arnowitt, Deser, and Misner. In 1959 Anderson reported that two particularly simple Hermitian orderings failed. ${ }^{13}$ [Actually Anderson reported "success" but his criterion for this did not include the requirement that the coefficient operators $C_{A B C}(p, q)$ of relation (1.3) stand to the left of the constraints $\phi_{C}(p, q)$. When this point is checked it is seen that both of Anderson's examples fail-as he was to note three years later. ${ }^{14}$ The search through more exotic arrangements reached an abrupt and unpleasant end in 1962 with the announcement, also by Anderson, that no Hermitian ordering worked. ${ }^{14}$ The very next year Schwinger contradicted this result by claiming consistency for the first of Anderson's 1959 examples. ${ }^{15}$ The discrepancy resulted from both author's heavy reliance upon distributional identities of the form

$$
\begin{aligned}
f(y) g(x) \delta^{\prime}(x-y)= & f(x) g(x) \delta^{\prime}(x-y) \\
& +f^{\prime}(x) g(x) \delta(x-y) .
\end{aligned}
$$

While this relation obviously works for well-behaved test functions, we will see in Sec. III that it fails for potentially singular canonical operator products and that one symptom of this failure is the ability to derive mutually inconsistent results such as those obtained by Anderson and Schwinger.

It is curious and unfortunate that neither author seems to have been aware of the other's work and that no one else thought the discrepancy worth understanding. (In a 1968 review lecture Dirac ${ }^{5}$ did take note of the fact that Schwinger's result suffered from the ambiguity associated with products of coincident $\delta$ functions. He did not, however, comment on Anderson's work or conclude that the problem was hopelessly ill defined in its unregulated form.) Indeed, the decade's only other original contribution to the factor-ordering problem was the suggestion by DeWitt in 1967 to ignore it altogether. ${ }^{16}$ DeWitt noted that since all ordering ambiguities are formally proportional to $\delta^{3}(0)$ and its derivatives, any dynamical effects from such terms can easily be segregated. Furthermore, if one assumes that these isolated terms can be separately regulated and renormalized, simple invariance considerations make it plausible that their net contribution to any renormalized physical result must be zero. Thus the whole program of field theory could be carried out as if the offending terms were never present in the first place. Put simply, DeWitt argued that $\delta^{3}(0)$ and its derivatives should be regarded as zero and this is, in fact, exactly what dimensional regularization does. ${ }^{17}$
The weakness of DeWitt's argument lies in his need to assume that ordering ambiguities can be renormalized away. This may not be possible. Or perhaps it can only be done for certain values of otherwise undetermined parameters of the theory. It is important to understand that the theory is no better off in the functional formalism than in the canonical. If one does not set $\delta^{3}(0)$ and its derivatives to zero in the functional formalism, then the factor ordering problem appears in three guises: (1) the functional measure; ${ }^{18}$ (2) order- $\hbar$ terms in the action; ${ }^{19}$ and (3) residual gauge dependence. It is not known how to deal with these matters any more than it is known how to order the canonical constraints. Claims to the contrary, for example, those by Fradkin and Velkovisky, ${ }^{18}$ are based on unregulated formal manipulations or noninvariant point splitting. Covariant techniques using dimensional regularization ignore the problem, they do not resolve it.

The last decade has witnessed a modest revival of interest in the factor-ordering problem, though without any improvement in the reliance upon singular- $\delta$ function identities. In 1975 Christodoulou claimed a result in the context of the functional formalism. ${ }^{20}$ Komar argued on general grounds that Hermiticity had to be abandoned $^{21}$ and in 1979 , exhibited a supposedly consistent non-Hermitian ordering ${ }^{22}$ which we will study later in Sec. III. More recently new claims of a resolution have been made by Christodoulakis and Zanelli. ${ }^{23}$ Undoubtedly more will come.

Given the incredible difficulties which already beset quantum gravity in the covariant formalism and the fact that dimensional regularization at least permits us to ignore the factor-ordering problem, it is debatable whether we should attempt a resolution at this time. On the other hand, the analogy with string theory suggests that Dirac consistency may imply drastic restrictions on how we can quantize gravity. Recent history ${ }^{20-23}$ makes it clear that the factor-ordering problem will continue to be studied. Given this, we feel that the effort should at least be made properly. In particular, the problem cannot even be meaningfully discussed until the singular operator products which invalidate previous formal manipulations are first regulated.

Our paper consists of six sections of which this Introduction is the first. Section II summarizes the gravitational canonical formalism and defines the problem. Section III explains why singular operator products make regulation essential. As an example we examine in detail the proof of Komar ${ }^{22}$ and show its inconsistency. Section IV treats the necessity of employing an invariant regulator and the problems with known techniques. In Sec. V we discuss the implications of our results for Ashtekar's new formulation of canonical quantum gravity. ${ }^{24}$ A brief conclusion comprises Sec. VI.

\section{DEFINITION OF THE FACTOR-ORDERING PROBLEM}

The fundamental classical field of pure gravity is the real, symmetric tensor $g_{\mu \nu}(x)$ with signature $(+---)$. Greek indices run from 0 to 3 while Latin ones run from 1 to 3 . The points $x$ comprise a four-dimensional space- 
time manifold ${ }^{(4)} M$. It will be assumed that the action functional $S[g]$ is invariant under one-to-one mappings which carry the coordinate label set of ${ }^{(4)} M$ onto itself. Although the conclusions of this paper apply to any coordinate-invariant metric (or vierbein) theory of gravity, we shall often use the Einstein-Hilbert action as a concrete example:

$S_{\mathrm{EH}}[g]=\frac{-1}{16 \pi G} \int_{(4)} d^{4} x\left[-{ }^{(4)} g(x)\right]^{1 / 2(4)} R(x)$.

Here $G$ is Newton's constant, ${ }^{(4)} R(x)$ is the Ricci scalar, and ${ }^{(4)} g(x)$ is the determinant of $g_{\mu \nu}(x)$. By varying $S_{\mathrm{EH}}[g]$ the usual Einstein equations emerge:

$$
\begin{aligned}
\frac{\delta S_{\mathrm{EH}}[g]}{\delta g_{\mu v}(x)}= & \frac{1}{16 \pi G}\left[{ }^{(4)} R^{\mu v}(x)-\frac{1}{2} g^{\mu v}(x)^{(4)} R(x)\right] \\
& \times\left[{ }_{-}^{(4)} g(x)\right]^{1 / 2}=0 .
\end{aligned}
$$

A well-defined initial-value problem requires eliminating local, time-dependent, general coordinate invariance. We shall do this by imposing the synchronous gauge

$$
g_{\mu 0}(x)=\eta_{\mu 0},
$$

where $\eta_{\mu v}$ is the Lorentz metric. There will generally be coordinate singularities in this gauge but they should not trouble invariant operators or invariant states. It is simple to show that any volume gauge condition such as (2.3) leaves a certain amount of residual coordinate invariance; for the synchronous gauge one has the freedom to perform time independent transformations of space and time. Infinitesimally the most general transformation of this type can be written as the product of two basic ones which we shall refer to as $C$ (for "3coordinate"):

$$
\delta_{C} X^{\mu} \equiv\left\{\begin{array}{l}
0 \quad \forall \mu=0, \\
\theta^{i}(\mathbf{x}) \quad \forall \mu \neq 0
\end{array}\right.
$$

and $T$ (for time)

$$
\delta_{T} X^{\mu} \equiv\left\{\begin{array}{l}
\tau(\mathbf{x}) \quad \forall \mu=0, \\
-\int_{0}^{x^{0}} d s g^{i j}(s, \mathbf{x}) \tau_{, j}(\mathbf{x}) \quad \forall \mu \neq 0 .
\end{array}\right.
$$

In these and all succeeding formulas a comma denotes differentiation and the Einstein summation convention is in force. It will be assumed that the coordinate label set of each constant-time, three-dimensional, spacelike surface $M$ in ${ }^{(4)} M$ is identical and topologically closed. This may impose constraints on the noninfinitesimal descendants of (2.4).

The canonical theory of classical gravity is formulated on any spacelike surface $M ;{ }^{10}$ we shall use $x^{0}=0$. The fundamental canonical field variables are the six $g_{i j}(\mathbf{x})$ and their conjugate momenta $p^{i j}(\mathbf{x})$ at each point $\mathbf{x} \in M$. These variables obey the usual Poisson-bracket relations

$$
\left\{g_{i j}(\mathbf{x}), p^{r s}(\mathbf{y})\right\}=\frac{1}{2}\left(\delta_{i}^{r} \delta_{j}^{s}+\delta_{i}^{s} \delta_{j}^{r}\right) \delta^{3}(\mathbf{x}-\mathbf{y}) .
$$

One determines the fields at anytime $x^{0}$ in terms of these initial values by evolving with the canonical Hamiltonian $H[p, g]$. Six of the Euler-Lagrange equations are thereby realized through the definition of how to time evolve the conjugate momenta

$$
\begin{aligned}
\frac{\delta S[g]}{\delta g_{i j}\left(x^{0}=0, \mathbf{x}\right)}= & -\frac{\partial}{\partial x^{0}} p^{i j}\left(x^{0}=0, \mathbf{x}\right) \\
& +\left\{p^{i j}(\mathbf{x}), H[p, g]\right\} \equiv 0 .
\end{aligned}
$$

The other four variations of the action,

$$
\begin{aligned}
& C_{i}(\mathbf{x}) \equiv 2 \frac{\delta S[g]}{\delta g^{0 i}\left(x^{0}=0, \mathbf{x}\right)}, \\
& T(\mathbf{x}) \equiv 2 \frac{\delta S[g]}{\delta g^{00}\left(x^{0}=0, \mathbf{x}\right)},
\end{aligned}
$$

do not vanish in this manner. A consequence of this fact is that not all initial-value field configurations $g_{i j}(\mathbf{x})$ and $p^{i j}(\mathbf{x})$ correspond to solutions of the theory. Since it is really the Euler-Lagrange equations which express the physics of gravitation, we must restrict attention to field configurations for which the constraints (2.7) vanish.

For Einstein's theory these constraints work out to be

$$
\begin{aligned}
C_{i}(\mathbf{x})=- & 2 g_{i j}(\mathbf{x}) p_{; k}^{j k}(\mathbf{x}) \\
T(\mathbf{x})=- & 16 \pi G \frac{1}{\sqrt{-g(\mathbf{x})}}\left[\frac{1}{2} g_{i j}(\mathbf{x}) g_{r s}(\mathbf{x})-g_{i r}(\mathbf{x}) g_{j s}(\mathbf{x})\right] \\
& \times p^{i j}(\mathbf{x}) p^{r s}(\mathbf{x})+\frac{1}{16 \pi G} \sqrt{-g(\mathbf{x})} R(\mathbf{x})
\end{aligned}
$$

[In these and all subsequent expressions we shall follow the usual canonical convention of working "covariantly" on the 3-surface $M$. Thus, the semicolon in (2.8a) refers to covariant differentiation on with respect to the affine connection formed from $g_{i j}(\mathbf{x}) ; R(\mathbf{x})$ is the corresponding Ricci scalar and $g(\mathbf{x})$ is the related determinant.] Whatever action is used, the constraints (2.7) generate the residual symmetry group (2.4) which is of course why we have been calling them $C_{i}$ and $T$. To be explicit, if we consider the integrated forms

$$
\begin{aligned}
& C[\boldsymbol{\theta}] \equiv \int_{M} d^{3} x \theta^{i}(\mathbf{x}) C_{i}(\mathbf{x}), \\
& T[\tau] \equiv \int_{M} d^{3} x \tau(\mathbf{x}) T(\mathbf{x}),
\end{aligned}
$$

and act them by Poisson bracket on a general functional of the dynamical variables $h[p, g]$

$$
\begin{aligned}
& \delta_{C} h[p, g]=\{C[\theta], h[p, g]\}, \\
& \delta_{T} h[p, g]=\{T[\tau], h[p, g]\},
\end{aligned}
$$

then the resulting quantities are the infinitesimal $C$ and $T$ transforms of $h[p, g]$. Applying this to the constraints themselves it is straightforward to show

$$
\begin{aligned}
& \left\{C\left[\boldsymbol{\theta}_{1}\right], C\left[\boldsymbol{\theta}_{2}\right]\right\}=C\left[\boldsymbol{\theta}_{1} \cdot \nabla \boldsymbol{\theta}_{2}-\boldsymbol{\theta}_{2} \cdot \nabla \boldsymbol{\theta}_{1}\right], \\
& \{C[\boldsymbol{\theta}], T[\tau]\}=T[\boldsymbol{\theta} \cdot \boldsymbol{\nabla} \tau], \\
& \left\{T\left[\tau_{1}\right], T\left[\tau_{2}\right]\right\}=C\left[\boldsymbol{\theta}_{12}\right], \\
& \theta_{12}^{i}(\mathbf{x}) \equiv g^{i j}(\mathbf{x})\left[\tau_{1, j}(\mathbf{x}) \tau_{2}(\mathbf{x})-\tau_{1}(\mathbf{x}) \tau_{2, j}(\mathbf{x})\right] .
\end{aligned}
$$


This is sometimes summarized by saying that the vector space of constraints forms an algebra under the multiplication defined by the Poisson bracket. Because the combination coefficients on the right-hand side are themselves dynamical quantities, the gravitational constraint algebra is said to be "open". However, we have been unable to resist spoiling the terminology slightly by referring to the fact that the constraints span an algebra as "closure." For our purposes it will often be more convenient to work with the constraint algebra in its unintegrated form

$$
\begin{aligned}
& \left\{C_{i}(\mathbf{x}), C_{j}(\mathbf{y})\right\}=\delta_{, i}^{3}(\mathbf{x}-\mathbf{y}) C_{j}(\mathbf{x})+\delta_{, j}^{3}(\mathbf{x}-\mathbf{y}) C_{i}(\mathbf{y}) \\
& \left\{C_{i}(\mathbf{x}), T(\mathbf{y})\right\}=\delta_{, i}^{3}(\mathbf{x}-\mathbf{y}) T(\mathbf{x}), \\
& \{T(\mathbf{x}), T(\mathbf{y})\}=-\delta_{, i}^{3}(\mathbf{x}-\mathbf{y})\left[g^{i j}(\mathbf{x}) C_{j}(\mathbf{x})+g^{i j}(\mathbf{y}) C_{j}(\mathbf{y})\right] .
\end{aligned}
$$

In the corresponding quantum field theory $g_{i j}(\mathbf{x})$ and $p^{i j}(\mathbf{x})$ become field operators which commute in the usual way:

$$
\left[g_{i j}(\mathbf{x}), p^{r s}(\mathbf{y})\right]=\frac{i}{2}\left(\delta_{i}^{r} \delta_{j}^{s}+\delta_{i}^{s} \delta_{j}^{r}\right) \delta^{3}(\mathbf{x}-\mathbf{y}) .
$$

We shall usually be able to avoid being very precise about the space of states $\Omega$ upon which they act, but the general idea is easy enough to sketch in a highly formal sense. States are represented by their wave functionals $\Psi[g] \equiv\langle g \mid \Psi\rangle$. The operators $g_{i j}(\mathbf{x})$ and $p^{i j}(\mathbf{x})$ act upon them by multiplication and functional differentiation, respectively. The inner product between two states is defined by the functional integral

$$
\begin{aligned}
\left\langle\Psi_{1} \mid \Psi_{2}\right\rangle \equiv \int & {[d p][d g] F[p, g] \widetilde{\Psi}_{1}^{*}[p] } \\
& \times \exp \left(i \int d^{3} x p^{i j}(\mathbf{x}) g_{i j}(\mathbf{x})\right) \Psi_{2}[g],
\end{aligned}
$$

$$
\widetilde{\Psi}[p] \equiv \int[d g] \exp \left[-i \int d^{3} x p^{i j}(\mathbf{x}) g_{i j}(\mathbf{x})\right) \Psi[g] \text {. }
$$

The quantity $F[p, g]$ represents the $T$ and $C$ gauge-fixing functionals and their associated Faddeev-Popov determinants. The functional measures are (very formally)

$$
\begin{aligned}
& {[d p] \sim \prod_{\mathbf{x} \in M} \prod_{i \leq j=1}^{3}\left(d^{3} x\right)^{1 / 3} d p^{i j}(\mathbf{x}) / 2 \pi,} \\
& {[d g] \sim \prod_{\mathbf{x} \in M} \prod_{i \leq j=1}^{3}\left(d^{3} x\right)^{2 / 3} d g_{i j}(\mathbf{x}) .}
\end{aligned}
$$

The space $\Omega$ consists of the completion of the set of states for which the norm given by (2.14) is finite. A consequence of the nonrenormalizability of Einstein's theory is that no one knows how to actually define this inner product, and hence $\Omega$, very precisely. For this reason we shall entirely avoid taking expectation values and seldom bother identifying the states upon which our operators act. Were we to announce a resolution to the factor-ordering problem these omissions would be damn- ing. As our result is negative, they merely permit us to study ambiguities of the problem which are independent of the space of states.

Along with $g_{i j}(\mathbf{x})$ and $p^{i j}(\mathbf{x})$, quantization promotes the constraints into operators. However, the precise arrangement of the various noncommuting factors which comprise them remains to be specified. Whatever convention is applied, it will not be the case that all states in $\Omega$ are annihilated by the constraints - any more that it was that all initial value field configurations obeyed the classical constraints. The set of states for which

$$
\begin{aligned}
& C_{i}(\mathbf{x})|\Psi\rangle=0, \\
& T(\mathbf{x})|\Psi\rangle=0,
\end{aligned}
$$

comprises the physical subspace $\Omega_{P}$. Since it is only on $\Omega_{P}$ that all the Euler-Lagrange equations are realized as operator relations, $\Omega_{P}$ is the proper arena for quantum gravity. The factor-ordering problem concerns whether we can devise a convention for ordering the constraint operators such that $\Omega_{P}$ has a chance of being nontrivial.

A constrained theory of canonical variables $p$ and $q$ is said to be Dirac consistent if its constraints $\phi_{A}(p, q)$ obey two conditions:

$$
\begin{aligned}
& {\left[\phi_{A}(p, q), \phi_{B}(p, q)\right]=C_{A B C}(p, q) \phi_{C}(p, q),} \\
& {\left[\phi_{A}(p, q), H[p, q]\right]=C_{A B}(p, q) \phi_{B}(p, q),}
\end{aligned}
$$

where the coefficient functions $C_{A B C}(p, q)$ and $C_{A B}(p, q)$ are nonsingular on the constrained subspace. The first condition is a requirement for the existence of nonzero states which are annihilated by the constraint operators. Note that the coefficient $C_{A B C}(p, q)$ must stand to the left of the constraint $\phi_{C}(p, q)$ in $(2.17 \mathrm{a})$. The second condition merely implies that the constrained subspace is preserved under time evolution. Since the Hamiltonian of quantum gravity is one of the constraints (recall that we are assuming $M$ to be compact) condition (2.17a) implies (2.17b). The failure of a general theory to be Dirac consistent either means that the set of constraints is not complete or that the physical subspace is limited to the state 0 . Because the constraints generate residual symmetries, with which it is inconceivable that quantum gravity is more richly endowed than its classical limit, we regard either situation as a disaster. The factorordering problem consists of specifying an ordering for the constraint operators so that this disaster is avoided.

\section{WHY THE PROBLEM IS ILL DEFINED}

From the classical constraint algebra (2.12) it is clear that the quantum result we are seeking is

$$
\begin{aligned}
& {\left[C_{j}(\mathbf{x}), C_{k}(\mathbf{y})\right]=i \delta_{, j}^{3}(\mathbf{x}-\mathbf{y}) C_{k}(\mathbf{x})+i \delta_{, k}^{3}(\mathbf{x}-\mathbf{y}) C_{j}(\mathbf{y}),} \\
& {\left[C_{j}(\mathbf{x}), T(\mathbf{y})\right]=i \delta_{, j}^{3}(\mathbf{x}-\mathbf{y}) T(\mathbf{x}),} \\
& {[T(\mathbf{x}), T(\mathbf{y})]=-i \delta_{, j}^{3}(\mathbf{x}-\mathbf{y})\left[g^{j k}(\mathbf{x}) C_{k}(\mathbf{x})+g^{j k}(\mathbf{y}) C_{k}(\mathbf{y})\right] .}
\end{aligned}
$$

Of course we are actually going to conclude that the 
whole problem is meaningless prior to regularization but our method of seeing this will be to proceed to a contradiction by following the usual, unregulated analysis. Since the $C$ constraints form a closed subalgebra we shall begin with them and for definiteness we may as well work with the Einstein theory.

From $(2.8 \mathrm{a})$ it is apparent that $C_{i}(\mathbf{x})$ is a sum of products of a single metric with a single momentum. Thus the extent of the ordering ambiguity is a single commutator. Whatever ordering we start with, we can always write the constraint as

$$
C_{i}(\mathbf{x})=-2\left[g_{i j}(\mathbf{x}) p^{j k}{ }_{, k}(\mathbf{x})+\Gamma_{i j k}(\mathbf{x}) p^{j k}(\mathbf{x})\right]+\alpha \delta_{, i}^{3}(0),
$$

where the number $\alpha$ depends upon the initial ordering. Now the appearance of an ill-defined quantity such as $\delta_{, i}^{3}(0)$ in (3.2) ought to give one pause. However, the usual statement at this point is that the final term is, after all, a $\mathbb{C}$ number, and so drops out of commutators. Thus it is asserted that the integrated $C$ constraints generate conventional $C$ transformations of operators, just as their classical ancestors did:

$$
\delta_{C} h[p, g]=-i[C[\theta], h[p, g]]
$$

This implies (3.1a) and (3.1b), no matter what the value of $\alpha$ is or how the $T$ operator is ordered. We feel there are grave problems with this line of reasoning. Arguing that a divergent constant has no effect on commutation relations is just the sort of thing that can fail when the theory is properly regulated. The reason is that the need to preserve symmetries may result in the "constant" becoming an operator in the regulated theory. We should also point out that same logic used to conclude that the $C-T$ commutator of quantum gravity agrees with its classical Poisson bracket also works for the $C-T$ commutator of bosonic string theory. Nonetheless it is precisely this commutator which harbors the anomaly of bosonic string theory. ${ }^{9}$ But as the issue stands out more clearly for the $T$ constraint we will face it there. Opinion is divided as to whether the parameter $\alpha$ should be determined by demanding "Hermiticity" (which gives $\alpha=-3$ ) or by requiring that $C[\theta]$ generates a $C$ transformation on states

$$
\delta_{c} \Psi[g] \equiv-i C[\theta] \Psi[g]=\int d^{3} x \delta_{c} g_{i j}(\mathbf{x}) \frac{\delta \Psi[g]}{\delta g_{i j}(\mathbf{x})}
$$

(which gives $\alpha=0$ ). Although both conditions are naively plausible, neither is very well defined. True Hermiticity is formulated in the context of an inner product such as (2.14) - which must remain highly formal until some sort of nonperturbative renormalizability is discovered for gravity. Nor does an equation such as (3.4) actually mean anything. The reason is that it can only be defined for smooth field configurations $g_{i j}(\mathbf{x})$ which form a set of measure zero in the usual function spaces of quantum field theory.

From $(2.8 b)$ it is apparent that $T(\mathbf{x})$ can be written as

$$
\begin{aligned}
T(\mathbf{x})= & -16 \pi G \frac{1}{\sqrt{-g}}\left(\frac{1}{2} g_{i j} g_{r s}-g_{i r} g_{j s}\right) p^{i j} p^{r s} \\
& +\frac{1}{16 \pi G} \sqrt{-g} R \\
& +\beta \delta^{3}(0) \frac{1}{\sqrt{-g}} g_{i j} p^{i j}+\gamma\left[\delta^{3}(0)\right]^{2} \frac{1}{\sqrt{-g}},
\end{aligned}
$$

where the $\mathbb{C}$ numbers $\beta$ and $\gamma$ parametrize the ordering. Because the constraint contains terms quadratic in the momenta there is no analog of (3.4) to determine these parameters:

$\delta_{T} \Psi[g] \equiv-i T[\tau] \Psi[g] \neq \int d^{3} x \delta_{T} g_{i j}(\mathbf{x}) \frac{\delta \Psi[g]}{\delta g_{i j}(\mathbf{x})}$

Nor does Hermiticity give a unique answer. Rather one must (attempt to) choose $\beta$ and $\gamma$ so as to enforce $(3.1 \mathrm{c})$. As (3.5) reveals, the $T$ constraint is even more poorly defined than the $C$ one. In addition to $\delta^{3}(0)$ it also contains the doubly meaningless expression $\left[\delta^{3}(0)\right]^{2}$. Furthermore, both quantities multiply operators and so do not drop out of commutation relations. Nevertheless, it might be argued that once a given ordering is selected we can absorb the $\beta$ and $\gamma$ terms by writing $T$ according to this convention. If the constraints are Dirac consistent then they act as the zero operator on a nontrivial physical space of states. Since zero is certainly a welldefined operator, the ill-defined expressions which result (supposing $\beta$ and $\gamma$ to be nonzero) from commuting all the $p$ 's to the right would be compensated by the fact that the reordered operator was similarly ill defined when acting on physical states. We shall not actually attack this argument, though we feel it to be incorrect. Instead we will demonstrate that it is not possible to realize its premise, a Dirac consistent ordering, without regularization.

To be more specific one can not prevent the appearance of ill-defined expressions in checking Dirac consistency. The reason is that distributional identities such as

$$
f(\mathbf{x}) g(\mathbf{y}) \delta^{3}(\mathbf{x}-\mathbf{y})=f(\mathbf{x}) g(\mathbf{x}) \delta^{3}(\mathbf{x}-\mathbf{y}),
$$

have to be invoked in order to assemble the various field operators which result from a typical constraint commutator back into more constraints. Such identities are valid enough when $f(\mathbf{x})$ and $g(\mathbf{x})$ are smooth test functions but ambiguities can arise when they become field operators. In particular if $f(\mathbf{x})$ and $g(\mathbf{y})$ commute to give another $\delta$ function (or a derivative of one) then (3.7) is hopelessly ill defined.

Before considering the phenomenon as it applies to the gravitational constraints we have found it useful to study it in the much simpler, albeit somewhat artificial context of a canonical scalar system in one spatial dimension:

$$
[\phi(x), \pi(y)]=i \delta(x-y) .
$$

The one-dimensional analog of (3.7) clearly implies 


$$
\begin{aligned}
\delta^{\prime}(x-y)[f(x) & g(x)+f(y) g(y)] \\
= & \delta^{\prime}(x-y)[f(y) g(x)+f(x) g(y)],
\end{aligned}
$$

for test functions $f(x)$ and $g(x)$. Recklessly applying this relation to the expression

$\delta^{\prime}(x-y)\left\{\phi(x)\left[2 \phi(x) \pi^{\prime}(x)+\phi^{\prime}(x) \pi(x)\right]+x \leftrightarrow y\right\}$

(which we might imagine as the result of commuting two unspecified constraints) we easily obtain an inconsistency. First employ (3.9) to shift the arguments of the leftmost $\phi$ 's:

$(3.10)=\delta^{\prime}(x-y)\left\{\phi(y)\left[2 \phi(x) \pi^{\prime}(x)+\phi^{\prime}(x) \pi(x)\right]+x \leftrightarrow y\right\}$.

Now commute these fields to the right using the identity

$$
\begin{aligned}
& {\left[\phi(y), 2 \phi(x) \pi^{\prime}(x)+\phi^{\prime}(x) \pi(x)\right]+x \leftrightarrow y} \\
& =2 i \phi(x) \delta^{\prime}(x-y)+i \phi^{\prime}(x) \delta(x-y)-2 i \phi(y) \delta^{\prime}(x-y) \\
& \quad+i \phi^{\prime}(y) \delta(x-y)=0 .
\end{aligned}
$$
ments of the (now) rightmost $\phi$ 's (at which point we have succeeded in reordering the original expression) and once to shift the arguments of the $\pi$ 's yield

$(3.10)=\delta^{\prime}(x-y)\left\{\left[2 \phi(x) \pi^{\prime}(y)+\phi^{\prime}(x) \pi(y)\right] \phi(x)+x \leftrightarrow y\right\}$.

However, starting from (3.10) we can use (3.9) to shift the arguments of the $\pi$ 's, giving

$(3.10)=\delta^{\prime}(x-y)\left\{\phi(x)\left[2 \phi(x) \pi^{\prime}(y)+\phi^{\prime}(x) \pi(y)\right]+x \leftrightarrow y\right\}$.
Two further applications of (3.9), once to shift the argu-

Upon subtracting (3.13) from (3.14) and simplifying, we are left with an expression of highly dubious mathematical pedigree

$$
\begin{aligned}
0=\delta^{\prime}(x-y)\left\{\left[\phi(x), 2 \phi(x) \pi^{\prime}(y)+\phi^{\prime}(x) \pi(y)\right]+x \leftrightarrow y\right\} \\
=\delta^{\prime}(x-y)\left\{-2 i \phi(x) \delta^{\prime}(x-y)+i \phi^{\prime}(x) \delta(x-y)\right. \\
\left.\quad+2 i \phi(y) \delta^{\prime}(x-y)+i \phi^{\prime}(y) \delta(x-y)\right\} \\
=\delta^{\prime}(x-y)\left[4 i \phi^{\prime}(x) \delta(x-y)\right] .
\end{aligned}
$$

While this argument might be proclaimed as a proof that $\delta(x) \delta^{\prime}(x)=0$, it seems obvious that (3.10) was ill defined to begin with and that our entirely formal manipulations have merely served to expose this.

Let us now return to quantum gravity. In 1979 Komar announced a proof of consistency for the following ordering: ${ }^{22}$

$$
\begin{aligned}
C_{i}= & -g_{i j}\left(p_{, k}^{j k}+\Gamma_{r s}^{j} p^{r s}\right)-\left(p_{, k}^{j k}+p^{r s} \Gamma_{r s}^{j}\right) g_{i j}, \\
T= & -16 \pi G(-g)^{-3 / 2} p^{i j}(-g)\left(\frac{1}{2} g_{i j} g_{r s}-g_{i r} g_{j s}\right) p^{r s} \\
& +\frac{1}{16 \pi G} \sqrt{-g} R .
\end{aligned}
$$

Though even the $C$ - $C$ commutator could be criticized on the general grounds we have raised, the argument becomes really compelling for the $T-T$ commutator. To get this Komar first defined

$$
K(\mathbf{x}) \equiv[-g(\mathbf{x})]^{3 / 2} T(\mathbf{x})
$$

and then wrote

$$
[T(\mathbf{x}), T(\mathbf{y})]=[-g(\mathbf{x})]^{-3 / 2}[-g(\mathbf{y})]^{-3 / 2}[K(\mathbf{x}), K(\mathbf{y})] .
$$

The $K-K$ commutator gives

$$
\begin{aligned}
-i[K(\mathbf{x}), K(\mathbf{y})]=-\frac{1}{2} \delta_{, i}^{3}(\mathbf{x}-\mathbf{y})\{[ & -g(\mathbf{x})]^{3} g^{i j}(\mathbf{x}) C_{j}(\mathbf{x})+C_{j}(\mathbf{x}) g^{i j}(\mathbf{x})[-g(\mathbf{x})]^{3} \\
& \left.+[-g(\mathbf{y})]^{3} g^{i j}(\mathbf{y}) C_{j}(\mathbf{y})+C_{j}(\mathbf{y}) g^{i j}(\mathbf{y})[-g(\mathbf{y})]^{3}\right\},
\end{aligned}
$$

and the problem reduces to commuting the second and fourth $C$ 's to the right. To accomplish this Komar applied (the three-dimensional analog of) (3.9) to the troublesome terms of (3.19):

$$
\delta_{, i}^{3}(\mathbf{x}-\mathbf{y})\left\{C_{j}(\mathbf{x}) g^{i j}(\mathbf{x})[-g(\mathbf{x})]^{3}+x \leftrightarrow y\right\}=\delta_{, i}^{3}(\mathbf{x}-\mathbf{y})\left\{C_{j}(\mathbf{y}) g^{i j}(\mathbf{x})[-g(\mathbf{x})]^{3}+x \leftrightarrow y\right\} .
$$

He then used the relation

$$
\left[[-g(\mathbf{x})]^{3} g^{j k}(\mathbf{x}), C_{k}(\mathbf{y})\right]=i \delta_{, k}^{3}(\mathbf{x}-\mathbf{y})\left\{[-g(\mathbf{x})]^{3} g^{j k}(\mathbf{x})+x \leftrightarrow y\right\},
$$

to perform the desired commutation

$$
\begin{aligned}
(3.20) & =\delta_{, i}^{3}(\mathbf{x}-\mathbf{y})\left\{g^{i j}(\mathbf{x})[-g(\mathbf{x})]^{3} C_{j}(\mathbf{y})+x \leftrightarrow y\right\} \\
& =\delta_{, i}^{3}(\mathbf{x}-\mathbf{y})\left\{g^{i j}(\mathbf{x})[-g(\mathbf{x})]^{3} C_{j}(\mathbf{x})+x \leftrightarrow y\right\} .
\end{aligned}
$$

Setting this back into (3.19) gives

$$
-i[K(\mathbf{x}), K(\mathbf{y})]=-\delta_{, i}^{3}(\mathbf{x}-\mathbf{y})\left\{[-g(\mathbf{x})]^{3} g^{i j}(\mathbf{x}) C_{j}(\mathbf{x})+x \leftrightarrow y\right\},
$$

whereupon substitution into (3.18) and a further application of (3.9) produces the desired result

$$
\begin{aligned}
-i[T(\mathbf{x}), T(\mathbf{y})] & =-\delta_{, i}^{3}(\mathbf{x}-\mathbf{y})\left[\left(\frac{g(\mathbf{x})}{g(\mathbf{y})}\right]^{3 / 2} g^{i j}(\mathbf{x}) C_{j}(\mathbf{x})+x \leftrightarrow y\right] \\
& =-\delta_{, i}^{3}(\mathbf{x}-\mathbf{y})\left[g^{i j}(\mathbf{x}) C_{j}(\mathbf{x})+x \leftrightarrow y\right] .
\end{aligned}
$$


As noted, the problem with such manipulations is that products of noncommuting operators (or any operators) do not constitute valid test functions. For example, in obtaining (3.20) it was necessary to use the distributional identity (3.9) with

$$
\begin{aligned}
& f \rightarrow C_{j}, \\
& g \rightarrow(-g)^{3} g^{i j} .
\end{aligned}
$$

By choosing $f$ and $g$ differently one can obtain contradictory results. For example, let us split the $C_{i}$ 's into sums of $g_{i j}-p^{r s}$ products and then apply (3.9) separately to each term with $f$ taken to be the $p^{r s}$ s. Instead of (3.20) we obtain

$$
\begin{array}{r}
\delta_{, i}^{3}(\mathbf{x}-\mathbf{y})\left\{C_{j}(\mathbf{x})[-g(\mathbf{x})]^{3} g^{i j}(\mathbf{x})+x \leftrightarrow y\right\}=-\delta_{, i}^{3}(\mathbf{x}-\mathbf{y})\left\{\left[g_{j k}(\mathbf{x}) p_{, l}^{k l}(\mathbf{y})+\Gamma_{j k l}(\mathbf{x}) p^{k l}(\mathbf{y})+p_{, l}^{k l}(\mathbf{y}) g_{j k}(\mathbf{x})\right.\right. \\
\left.\left.+p^{k l}(\mathbf{y}) \Gamma_{j k l}(\mathbf{x})\right][-g(\mathbf{x})]^{3} g^{i j}(\mathbf{x})+x \leftrightarrow y\right\}
\end{array}
$$

After many tedious commutations and applications of (3.7) and (3.9) this can be written as

$$
(3.26)=\delta_{, i}^{3}(\mathbf{x}-\mathbf{y})\left\{[-g(\mathbf{x})]^{3} g^{i j}(\mathbf{x}) C_{j}(\mathbf{x})+x \leftrightarrow y\right\}-i \delta^{3}(\mathbf{x}-\mathbf{y}) \delta_{, i}^{3}(\mathbf{x}-\mathbf{y})[-g(\mathbf{x})]^{3}\left[5 g^{i j}{ }_{, j}(\mathbf{x})+g^{i j}(\mathbf{x}) g^{k l}(\mathbf{x}) g_{k l, j}(\mathbf{x})\right]
$$

Hence, Komar's $K-K$ commutator is equal to

$$
\begin{aligned}
-i[K(\mathbf{x}), K(\mathbf{y})]= & -\delta_{, i}^{3}(\mathbf{x}-\mathbf{y})\left\{[-g(\mathbf{x})]^{3} g^{i j}(\mathbf{x}) C_{j}(\mathbf{x})+x \leftrightarrow y\right\} \\
& +\frac{i}{2} \delta^{3}(\mathbf{x}-\mathbf{y}) \delta_{, i}^{3}(\mathbf{x}-\mathbf{y})[-g(\mathbf{x})]^{3}\left[5 g_{, j}^{i j}(\mathbf{x})+g^{i j}(\mathbf{x}) g^{k l}(\mathbf{x}) g_{k l, j}(\mathbf{x})\right]
\end{aligned}
$$

The first term is Komar's result (3.23) so we have just "proved" that the second term vanishes. Except, of course, that the second term, and hence the whole expression, is ill defined by virtue of the fact that it contains a product of coincident $\delta$ functions.

Let us conclude by reviewing the logic. In order to prove Dirac consistency it is necessary to employ $\delta$ function identities such as (3.7) and its derivatives for test functions which include products of noncommuting operators. Once this is asserted to be a valid step one can use the identities differently to introduce meaningless distributional products [like $\delta(x) \delta^{\prime}(x)$ ] and produce contradictory results. The ability to access meaningless expressions and derive contradictory results by seemingly legitimate applications of the stated (and in this case necessary) rules is the hallmark of an ill-defined system. Thus we conclude that the factor-ordering problem is ill defined.

\section{REMARKS ON REGULARIZATION}

The problems of the previous section obviously derive from the need to use distribution theory identities such as (3.7) with "test functions" which include ill-defined products of noncommuting, local operators. Two points deserve additional notice. First, problems with operator products are not specific to quantum gravity. Thus one might hope that techniques which work in other quantum field theories can be applied to the gravitational constraint algebra. Second, even commuting operators generally have singular products in the presence of physically interesting states. Our concentration in Sec. III on noncommuting products was only to avoid having to actually produce a physically interesting state for quantum gravity.

Both facts are sufficiently illustrated by considering the operator $\phi^{2}(x)$ in free scalar field theory. In the same spirit that we might argue the $C_{i}(\mathbf{x})$ constraints are well defined with $\alpha=0$ (because they act on wavefunctionals by infinitesimally transforming the metric) so too we would have to accept that $\phi^{2}(x)$ makes sense. After all it acts by simple multiplication in the position representation. Yet the vacuum expectation value of this operator is better known as the coincidence limit of the free propagator and it diverges in two or more dimensions. Thus even the nicest possible operator product, with no noncommuting factors, in the nicest possible theory, acts upon the simplest possible state of that theory in an indisputably singular manner.

The general procedure for dealing with ill-defined expressions in quantum field theory is regularization and this is obviously what should be done with the gravitational constraint algebra. First regulate the theory to make sense of all operator products, then attempt to enforce Dirac consistency. It is clearly simplest to imagine doing this with an invariant regulator; a little thought leads one to the conclusion that it is also necessary. To see this, note that the closure of the gravitational constraints is a consequence of general coordinate invariance. Thus the regulated constraints are not expected to close with a noninvariant regulator. For renormalizable theories this is no more than a nuisance. One merely renormalizes the operators under study and then attempts to enforce Dirac consistency in the now well-defined unregulated limit. For the Einstein theory we do not know how to renormalize so there is no unregulated limit. The only sense in which we can study Dirac consistency for the Einstein theory is with the regulator on. But the regulated constraints do not close-even classicallywhen the regulator does not respect general coordinate invariance. Hence an invariant regulator must be used.

Unfortunately no known regularization scheme is both invariant and capable of removing the sort of divergences one encounters in studying the canonical con- 
straint algebra. Dimensional regularization is sometimes held up as a counter example but this is incorrect. It is of course somewhat difficult to envisage the method in a strong operator sense-what does the metric mean for a nonintegral number of dimensions?-but even in the weak sense there is a problem. The issue is how to interprete the various $\delta^{D-1}(0)$ terms which are routinely produced in reordering products of coincident operators. One argues that these are zero in dimensional regularization by first introducing a mass, computing the desired inner product, and then letting the mass vanish. ${ }^{17}$ This procedure obviously breaks general coordinate invariance and one is left to worry about potential anomalies. Presumably these would arise in the covariant formalism as violations of the Ward identities associated with changing residual gauge conditions $[F[p, g]$ in expression (2.14a)]. Their absence, which has never been shown, is equivalent to obtaining a consistent canonical factor ordering.

Other regulators are similarly unsatisfactory. The $\zeta$ function method does not really eliminate the necessary divergences. To be more explicit, it will produce an effective action from which finite Green's functions can be computed as long as the fields are evaluated at different points. However, as soon as the points are allowed to coincide, which must happen to form nonlinear constraints, divergent expressions result. Pauli-Villars regularization can be made invariant through the use of higher derivative actions but the resulting canonical theory has a fundamentally different structure from the original one. Furthermore, products of coincident operators are still singular, just as with the $\zeta$-function method.

Point splitting certainly regulates everything but it is very hard to formulate invariantly. The reason is that general coordinate invariance requires that the separated points have a geometrically meaningful relation to one another. For example, we might require that they be joined by geodesics. This is easy enough to do with respect to a given background geometry, but it is of course the full quantum geometry which must be used. One then has to entertain the notion of evaluating operators at coordinates (the geodesic endpoints) which are themselves operators. In itself this is not fatal, it at least makes sense in perturbation theory - the real problem is that any geometrically meaningful separation scheme itself introduces products of local operators. It might be thought that point splitting can at least be used to regulate products of noncommuting operators in a $C$ invariant (but not $T$-invariant) fashion. However, even this is false, although the reason is rather subtle. Since the point is potentially significant we shall go through the argument.

Define the geodesic operator by the equations

$$
\begin{aligned}
& \ddot{\chi}^{i}(t)+\Gamma_{j k}^{i}[\chi(t)] \dot{\chi}^{j}(t) \dot{\chi}(t)=0, \\
& \chi^{i}(0)=\chi^{i}, \\
& \dot{\chi}^{i}(0)=e_{a}^{i}(\mathbf{x}) V^{a} .
\end{aligned}
$$

Here $e_{a}^{i}$ is the vierbein field operator on $M$ :

$$
g^{i j}(\mathbf{x}) \equiv e_{a}^{i}(\mathbf{x}) e_{b}^{j}(\mathbf{x}) \eta^{a b}
$$

and the Lorentz index $a$ runs from 1 to 3 . Parallel transport is accomplished by means of the operator

$$
M_{j}^{i}(V, X) \equiv P\left[\exp \left(+\int_{0}^{1} d t \dot{\chi}^{k}(1-t) \Gamma_{k j}^{i}[\chi(1-t)]\right)\right],
$$

where the symbol $P$ denotes path ordering. The putative regularization method consists of replacing the canonical momenta of the operator under study with the smeared expression

$$
P^{i j}(\mathbf{x}) \rightarrow P_{\epsilon}^{i j}(\mathbf{x}) \equiv\left(2 \pi \epsilon^{2}\right)^{-3 / 2} \int d^{3} V \exp \left[\frac{1}{2 \epsilon^{2}} V^{a} V^{b} \eta_{a b}\right) M_{k}^{i} M_{l}^{j} p^{k l}[\chi(1)] .
$$

One does nothing to the $g_{i j}(\mathbf{x})$ 's since parallel transport would carry them into themselves anyway.

The analog of (3.2),

$$
C_{i}(\mathbf{x}, \epsilon) \equiv-2\left[g_{i j}(\mathbf{x}) P_{\epsilon}^{j k}(\mathbf{x})\right]_{, k}+g_{j k, i}(\mathbf{x}) P_{\epsilon}^{j k}(\mathbf{x})+\frac{\alpha}{12}\left\{-2\left[g_{i j}(\mathbf{x}), P_{\epsilon}^{j k}(\mathbf{x})\right]_{, k}+\left[g_{j k, i}(\mathbf{x}), P_{\epsilon}^{j k}(\mathbf{x})\right]\right\}
$$

is well defined, at least as regards products of coincident, noncommuting operators. On the other hand, the commutator of two such constraints no longer gives a third constraint. The reason has nothing to do with ordering; even the classical Poisson bracket of two $C_{i}(\mathbf{x}, \epsilon)$ 's is not resolvable into more $C_{i}(\mathbf{x}, \epsilon)$ 's. The real problem is that the theory is still $C$ invariant but the regulated constraints do not generate the symmetry. Therefore, they do not close. Indeed we would not want them to do so since this would imply the existence of a new symmetry. To find the true $C$ generators one must regulate the gauge-fixed action and apply Noether's theorem. This produces an unpleasant result: the old $C$ constraints.

Actually we should have seen this coming. As long as the theory is based on the fields $g_{i j}(\mathbf{x})$ and $p^{i j}(\mathbf{x})$ and is
$C$ invariant, the old $C$ constraints will always generate the symmetry. It follows that what we really want in a regulated theory is fewer degrees of freedom and a different symmetry group which only approaches general coordinate invariance in the unregulated limit. The ideal procedure for regulating operator products is to reduce the theory to a finite quantum mechanical system. Latticization is the epitome of such methods; an ultraviolet cutoff in a finite spacetime box is another example. In such a system one could actually do something, freed from the crippling restriction of not even being able to take an inner product. Of course the catch is finding a suitable symmetry group but this may not be as impossible as it seems. Recently one of us (R.P.W.) has had success in discretizing the Virasoro algebra of bo- 
sonic string theory. ${ }^{25}$ As the two constraint algebras are closely related one might hope to extend the method to canonical quantum gravity.

\section{NEW VARIABLES}

It is important to understand that we have been discussing a very general problem of unregulated, local constraint algebras. The basic problem is that typical constraint commutators do not automatically assemble themselves into more constraints. Instead it is almost always necessary to shift the arguments of certain fields from $x$ to $y$ and vice versa using identity (3.7) or one of its descendants. Once this is allowed the cataract of disasters (3.10)-(3.15) cannot be avoided. A corollary is that no change of variables can possibly eliminate the need to regulate the constraints of quantum gravity before studying Dirac consistency.

As an example consider the spinorial variables recently proposed by Ashtekar, ${ }^{24}$ whose notation we shall follow. Of course Ashtekar was very careful not to claim a proof of Dirac consistency because of the problems we have been discussing, but suppose we disregard his caveat. The analog of $(3.1 \mathrm{c})$ for the spinorial system is

$$
\frac{2}{\hbar^{2}}\left[\operatorname{Tr}\left[\hat{\sigma}^{a}(x) \widehat{\sigma}^{b}(x)^{ \pm} \hat{F}_{a b}(x)\right], \operatorname{Tr}\left[\hat{\sigma}^{c}(y) \hat{\sigma}^{d}(y)^{ \pm} \hat{F}_{c d}(y)\right]\right]=\frac{2 \sqrt{2}}{\hbar} \delta_{, a}(x-y)\left\{\hat{q}^{a b}(x) \operatorname{Tr}\left[\hat{\sigma}^{c}(x)^{ \pm} \hat{F}_{b c}(x)\right]+x \leftrightarrow y\right\}
$$

It is tedious but straightforward to check that this follows from naive commutation, without the need to move any of the $\hat{q}^{a b}$ 's to the left of the $C$ constraint $\operatorname{Tr}\left(\hat{\sigma}^{c \pm} \hat{F}_{b c}\right)$. One might thus argue, as Ashtekar was careful not to do, that this result is more valid than Komar's. That such an argument would be nonsense follows from the fact that one still has to use (3.7) [in its derivative form (3.9)] in deriving (5.1). It was this, not the need for reorderings, that invalidated Komar's proof.

In fact the very same ambiguity we found in (3.15) is easy to obtain for the spinorial system. Consider a single one of the many terms on the right-hand side of (5.1):

$\delta_{, a}(x-y)\left\{\hat{q}^{a b}(x) \operatorname{Tr}\left[\hat{\sigma}^{c}(x)^{ \pm} \hat{A}_{c, b}(x)\right]+x \leftrightarrow y\right\}$.

If (3.9) is regarded as valid - and we remind the reader that this is the only way to derive $(5.1)$ - then we can rewrite this as

$$
(5.2)=\delta_{, a}(x-y)\left\{\hat{q}^{a b}(y) \operatorname{Tr}\left[\hat{\sigma}^{c}(x)^{ \pm} \hat{A}_{c, b}(x)\right]+x \leftrightarrow y\right\} .
$$

Commuting the $\hat{q}^{a b}$ 's to the left, inside the bracketed expression, gives zero:

$$
\begin{aligned}
& {\left[\hat{q}^{a b}(y), \operatorname{Tr}\left[\hat{\sigma}^{c}(x)^{ \pm} \hat{A}_{c, b}(x)\right]\right]+x \leftrightarrow y} \\
& \quad=\frac{\hbar}{\sqrt{2}} \delta_{, b}(x-y) \operatorname{Tr}\left[\hat{\sigma}^{b}(y) \hat{\sigma}^{a}(x)+\hat{\sigma}^{a}(y) \hat{\sigma}^{b}(x)\right] \\
& \quad-\frac{\hbar}{\sqrt{2}} \delta_{, b}(x-y) \operatorname{Tr}\left[\hat{\sigma}^{b}(x) \hat{\sigma}^{a}(y)+\hat{\sigma}^{a}(x) \hat{\sigma}^{b}(y)\right]=0 .
\end{aligned}
$$

Hence we have

$$
(5.2)=\delta_{, a}(x-y)\left\{\operatorname{Tr}\left[\hat{\sigma}^{c}(x)^{ \pm} \widehat{A}_{c, b}(x)\right] \widehat{q}^{a b}(y)+x \leftrightarrow y\right\} .
$$

Two further applications of (3.9) result in the expression

$$
(5.2)=\delta_{, a}(x-y)\left\{\operatorname{Tr}\left[\hat{\sigma}^{c}(x)^{ \pm} \hat{A}_{c, b}(y)\right] \hat{q}^{a b}(x)+x \leftrightarrow y\right\} .
$$

However, by applying (3.9) differently to (5.2) we obtain

$$
(5.2)=\delta_{, a}(x-y)\left\{\hat{q}^{a b}(x) \operatorname{Tr}\left[\hat{\sigma}^{c}(x)^{ \pm} \widehat{A}_{c, b}(y)\right]+x \leftrightarrow y\right\} .
$$

Subtracting the two versions gives the same sort of paradox that we encountered in expression (3.15):

$$
\begin{array}{r}
0=\delta_{, a}(x-y)\left[\sqrt{2} \hbar \hat{q}^{a b}(x) \delta_{, b}(x-y)\right. \\
\left.-\sqrt{2} \hbar \hat{q}^{a b}(y) \delta_{, b}(x-y)\right] \\
=\delta_{, a}(x-y)\left[-\sqrt{2}_{\hat{q}}{ }^{a b}{ }_{, b}(x) \delta(x-y)\right] .
\end{array}
$$

Thus expression (5.1) is not one bit better defined than the analogous result claimed by Komar.

It is perhaps worth pointing out that Ashtekar integrated his constraints up against smearing functions while we have used the unintegrated form. This in no way weakens our objection. The problem with using distributional identities such as (3.7) and its descendants up against local operators is that products of noncommuting operators are not valid test functions. Multiplying the whole ill-defined expression by a smooth, nonzero function and integrating does not change this. To obtain a well-defined expression one would have to separately smear each operator in the product first, before using the product as a test function. This can be done of course-it amounts to a noninvariant form of point splitting - but then general coordinate invariance is lost and the constraints consequently fail to close.

We conclude that no change of variables, Ashtekar's included, can eliminate the need for regulating the constraints of quantum gravity. However, it does not follow that changing variables cannot make the theory more amenable to regularization and here Ashtekar's system may have an advantage. In particular it has been suggested that the SU(2) structure of Ashtekar's system permits a natural lattice formulation. We refer the reader to the so far unsuccessful but ongoing work of Renteln and Smolin. ${ }^{26}$

\section{CONCLUSIONS}

We have shown how the constraints of quantum gravity are hopelessly ill defined prior to regularization. The 
problem is that examining Dirac consistency inevitably involves using distributional identities such as (3.7) for test functions which include products of noncommuting operators. The ambiguities thus engendered are responsible for the contradictory claims which have appeared in the literature. ${ }^{14,15,22}$ Though we illustrated the problem using the $T-T$ commutator, the $C-T$ and $C-C$ commutators are also poorly defined and further problems may exist because of products of commuting operators which act singularly on physically interesting states. Finally, no change of variables can remove the need for regularization.

Because the Einstein theory is nonrenormalizable its unregulated limit is not presently definable (though one might hope for some sort of nonperturbative renormalizability in the future). Thus the only context in which one can currently study the Dirac consistency of quantum gravity is a regulated theory with the regulator on. This requires general coordinate invariance or some ana$\log$ of it. Existing methods either fail to regulate products of coincident operators or else sacrifice general coordinate invariance in doing so. The most hopeful approach seems to be reducing to a finite-dimensional system (for example a lattice) which possesses a symmetry group that plausibly approaches general coordinate invariance in the (ill-defined) continuum limit.

Because ours is basically negative result we wish to stress again that the problem of whether or not quantum gravity is Dirac consistent is both real and potentially important. A definitive answer must reach one of the following conclusions.

(1) The gravitational constraints are Dirac consistent for any choice of spacetime dimension, coupling constants, and matter couplings.

(2) Dirac consistency follows only for certain values of these parameters.

(3) No choice of parameters gives Dirac consistency.

Any of these results would be exciting, (2) most of all. We stress again that this sort of thing can happen; obtaining $D=26$ for the spacetime dimension of bosonic string theory is an example. ${ }^{9}$

Note added in proof. After this work was completed we were apprised of a paper by Friedman and Jack in which many of the same results are independently obtained. ${ }^{27}$

\section{ACKNOWLEDGMENTS}

We have profited from conversations with A. Ashtekar, T. Jacobson, A. Komar, K. Kuchař, and P. Renteln. This work was supported in part by NSF Grants Nos. PHY83-10654, PHY83-18635, and PHY85-06686.
"Present address: Department of Physics, Brown University, Providence, RI 02912.

${ }^{1}$ G. 't Hooft and M. Veltman, Ann. Inst. Henri Poincaré 20A, 69 (1974).

${ }^{2}$ A. Sandage, Observatory 88, 91 (1968).

${ }^{3}$ G. 't Hooft, Nucl. Phys. B33, 173 (1971); C. G. Bollini and J. J. Giambiagi, Phys. Lett. 40B, 566 (1972); G. 't Hooft and M. Veltman, Nucl. Phys. B44, 189 (1972).

${ }^{4}$ B. S. DeWitt, Dynamical Theory of Groups and Fields (Gordon and Breach, New York, 1965); Phys. Rev. 162, 1195 (1967); 162, 1239 (1967).

${ }^{5}$ P. A. M. Dirac, Lectures on Quantum Mechanics (Yeshiva University, New York, 1964); Contemporary Physics (IAEA, Vienna, 1969).

6P. A. M. Dirac, Can. J. Math. 2, 129 (1950); Proc. R. Soc. London A246, 326 (1958).

${ }^{7}$ J. L. Anderson and P. G. Bergmann, Phys. Rev. 83, 1018 (1951).

${ }^{8}$ P. A. M. Dirac, Rev. Mod. Phys. 34, 592 (1962); J. S. Schwinger, Phys. Rev. 127, 324 (1962); 130, 406 (1962); 130, 800 (1962); 130, 1253 (1962).

${ }^{9}$ M. B. Green, J. H. Schwarz, and E. Witten, Superstring Theory I (Cambridge University Press, London, 1987).

${ }^{10}$ P. A. M. Dirac, Proc. R. Soc. London A246, 333 (1958).

${ }^{11}$ P. A. M. Dirac, Phys. Rev. 114, 924 (1959).

${ }^{12}$ R. Arnowitt and S. Deser, Phys. Rev. 113, 745 (1959); R. Arnowitt, S. Deser, and C. W. Misner, ibid. 116, 1322 (1959).

13J. L. Anderson, Phys. Rev. 114, 1182 (1959).
${ }^{14} \mathrm{~J}$. L. Anderson, in Proceedings of the First Eastern Theoretical Physics Conference 1962, edited by M. E. Rose (Gordon and Breach, New York, 1963).

15J. Schwinger, Phys. Rev. 132, 1317 (1963).

${ }^{16}$ B. S. DeWitt, Phys. Rev. 160, 1113 (1967).

${ }^{17}$ D. M. Capper and G. Leibbrandt, J. Math. Phys. 15, 82 (1974); 15, 86 (1974).

${ }^{18}$ E. S. Fradkin and G. Velkovisky, Phys. Rev. D 8, 4241 (1973).

${ }^{19}$ J. R. Klauder, in Field Theory and Strong Interactions, proceedings of the XIX Internationale Universitätswochen für Kernphysik, Schladming, edited by P. Urban (Acta Phys. Austriaca Suppl. 22) (Springer, Vienna, 1980).

${ }^{20}$ D. Christodoulou, in Proceedings of the First Marcel Grossman Meeting, Trieste 1975, edited by R. Ruffini (NorthHolland, Amsterdam, 1977).

${ }^{21}$ A. Komar, Phys. Rev. D 19, 2908 (1979).

${ }^{22}$ A. Komar, Phys. Rev. D 20, 830 (1979).

${ }^{23}$ T. Christodoulakis and J. Zanelli, Nuovo Cimento 93B, 1 (1986); 93B, 22 (1986).

${ }^{24}$ A. Ashtekar, Phys. Rev. Lett. 57, 2244 (1986).

${ }^{25}$ M. Srednicki and R. P. Woodard, Phys. Lett. B 196, 55 (1987).

${ }^{26}$ P. Renteln and L. Smolin, ITP Report No. 86-137, 1986 (unpublished).

${ }^{27}$ J. L. Friedman and I. Jack, University of Wisconsin report, 1987 (unpublished). 